\title{
Sunburnt fission yeast
}

\section{Andrew W. Murray}

ON page 368 of this issue ${ }^{1}$, Walworth and colleagues offer insights into the feedback control that prevents cells with damaged DNA from entering mitosis. They have identified a gene (termed $c h k l$, for checkpoint) which interacts both with $c d c 2$, a gene that encodes the protein whose activation induces mitosis, and with genes whose products are involved in the detection of DNA damage. Their observations provide strong support for the idea that damaged DNA prevents entry into mitosis by controlling the activity of $\mathrm{Cdc} 2$ protein.

Passage through the G2/M checkpoint of the cell cycle is induced by the activation of MPF (maturation promoting factor), a protein kinase that is composed of $\mathrm{Cdc} 2$ and cyclin B. The feedback control that regulates passage through this checkpoint must consist of three sets of components: those that detect damaged or unreplicated DNA, a signalling pathway activated by these lesions, and enzymes that the signalling pathway regulates to control the activation of MPF (reviewed in refs 2 and 3 ).

Genetic analysis of the fission yeast has revealed two classes of mutants that allow cells with damaged or unreplicated DNA to enter mitosis. Members of the first class have no effect on the size at which undamaged cells activate MPF and enter mitosis, but prevent either unreplicated or damaged DNA from arresting cells in G2. These mutations - which include $\mathrm{radl}^{-}$, $\mathrm{rad3}^{-}, \mathrm{rad} 9^{-}, \mathrm{rad} 17^{-}$(sensitive to radiation) and us I $^{-}$(sensitive to hydroxyurea, an inhibitor of DNA synthesis) - presumably identify mutants involved in the detection of DNA lesions and the transmission of this information to the cellcycle engine $^{4-6}$.

The second class of mutations disrupt the normal regulation of MPF activation and have two effects: they reduce the minimal size that cells must reach before they can enter mitosis and they abolish the ability of unreplicated DNA to prevent entry into mitosis. Most of these mutations affect the activity of the protein kinases and phosphatases that modify tyrosine 15 of $\mathrm{Cdc} 2$, a residue whose phosphorylation inhibits MPF activity. For example, overexpression of $\mathrm{Cdc} 25$, the tyrosine phosphatase, reduces cell size and allows cells with unreplicated DNA (but not those with damaged DNA) to enter mitosis ${ }^{7,8}$. Inactivation of the two partially redundant protein kinases, Wee1 and Mik1, that are responsible for phosphorylating this residue, induces mitotic catastrophe: even when DNA replication is not inhibited cells enter mitosis at such a small size that they die, and inhibition of DNA replication does not block entry into mitosis $^{8}$. The molecular details of the mechanism by which unreplicated DNA modulates the tyrosine phosphorylation of $\mathrm{Cdc} 2$ are unknown. Although an earlier report suggested that Wee1-dependent phosphorylation of $\mathrm{Cdc} 2$ was required for radiation-induced $\mathrm{G} 2$ arrest $^{9}$, more recent work demonstrates that $\Delta$ weel strains can still arrest in response to DNA damage (A. M. Carr, personal communication).

The lethal consequences of simultaneously inactivating Wee1 and Mik1 can be overcome by mutations in $c d c 2$, and it was during the investigation of one of these mutations that Walworth et al. ${ }^{1}$ discovered a gene involved in the response to damaged DNA. They isolated highcopy suppressors of the cold-sensitive growth of a $c d c 2^{c s}$ mutant and showed that one of these, $\operatorname{chkl}$, encodes a putative protein kinase. Deletion of $c h k l$ produced cells that grew normally but were sensitive to ultraviolet irradiation and failed to delay entry into mitosis when they were irradiated in G2. The mutant cells also fail to protect themselves against DNA damage caused by a temperature-sensitive mutation in DNA ligase. In contrast, the deletion mutants are not sensitive to hydroxyurea, suggesting that $\operatorname{chkl}$ is not involved in the response to unreplicated DNA. As Walworth et al. point out, it is hard to understand why a gene whose overexpression appears to stimulate MPF activity should also be a component of the machinery required to inhibit the entry into mitosis in response to damaged DNA.

Mutations in $\mathrm{Cdc} 2$ and the genes that regulate its activity seem to affect the response to either unreplicated DNA or damaged DNA but not both. Thus the dominant $c d c 2-3 w$ mutation allows cells with unreplicated DNA to enter mitosis ${ }^{6}$ but has no effect on the response to DNA damage $^{7}$, whereas $\Delta c h k l$ cells have exactly the opposite phenotype ${ }^{1}$. The phenotype of the $c d c 2-3 w$ and $\Delta c h k 1$ mutations is considerably less severe than that of the $\mathrm{rad}^{-}$and $\mathrm{hus}^{-}$mutations that inactivate the mitotic feedback control: $\mathrm{radl}^{-}$cells are more sensitive to irradiation than $\Delta c h k l$ cells and more sensitive to hydroxyurea than $c d c 2-3 w$ cells $^{1,4,5}$. Mutations in husl cause cells to die well before they enter mitosis ${ }^{6}$, implying that this gene is required to maintain the viability of interphase cells whose DNA replication has been inhibited, as well as preventing such cells from entering mitosis.

In mammalian cells DNA damage blocks the firing of replication origins until the lesions have been repaired ${ }^{10}$, suggesting that the Rad and Hus gene products may regulate the initiation of DNA rep- lication in fission yeast. In addition, the feedback controls in fission yeast may regulate more than one step in the induction of mitosis. In budding yeast, mutations that prevent the tyrosine phosphorylation of $\mathrm{Cdc} 28$ (the homologue of $\mathrm{Cdc} 2$ ) do not abolish the response to unreplicated DNA, suggesting that some other steps in the induction of mitosis must be regulated by feedback controls ${ }^{11,12}$.

Mutations that affect the response to damaged DNA have now been identified in both budding ${ }^{13,14}$ and fission yeast. None of the genes in one yeast has yet been shown to be homologous to those in the other, and the effect of preventing tyrosine phosphorylation of Cdc2 is different from that of preventing tyrosine phosphorylation of $\mathrm{Cdc} 28$. Although these discrepancies might mean that the feedback control that responds to damaged DNA is fundamentally different in these two organisms, I favour the possibility that, like most other parts of the cell cycle, the response to DNA damage has been conserved throughout evolution, albeit with some variation between organisms with different life styles.

This issue may be of more than academic interest. Mutations in the mammalian $p 53$ gene, which play a central role in human cancer, inactivate a feedback control that prevents cells with damaged DNA from entering $S$ phase ${ }^{15}$. In patients with the inherited recessive disorder, ataxia telangiectasia, levels of p53 do not increase in response to DNA damage $^{16}$, implying that these patients' cells cannot detect damaged DNA. Perhaps some of the budding and fission yeast genes, whose products prevent cells with damaged and unreplicated DNA from entering mitosis, will turn out to have homologues in mammalian cells whose inactivation is involved in the genetic stability seen in human tumours.

Andrew W. Murray is in the Departments of Physiology, and Biochemistry and Biophysics, University of California at San Francisco, San Francisco, California 94143, USA

\footnotetext{
1. Walworth, N., Davey, S. \& Beach, D. Nature 363. 368-371 (1993).

2. Hartwell, L. H. \& Weinert, T. A. Science 246, 629-634 (1989).

3. Murray, A. W. Nature 359, 599-604 (1992)

4. Al-Khodairy, F. \& Carr, A. M. EMBO J. 11, 1343-1350 (1992).

5. Rowley, R., Subramani, S. \&Young, P. G. EMBO J. 11, 1335-1342 (1992)

6. Enoch, T., Carr, A. M. \& Nurse, P. Genes Dev. 6. 2035-2046 (1992)

7. Enoch, T. \& Nurse, P Cell60,665-673 (1990) 8. Lundgren, K. etal. Cell64, 1111-1122 (1991) 9. Rowley, R., Hudson, J. \& Young. P. G. Nature 356 353-355 (1992)

10. Painter, R. B. \& Young, B. R. Radiat. Res. 64, 648-656 (1975).

11. Sorger, P. K. \& Murray, A. W. Nature 355, 365-368 (1992).

12. Amon, A. etal. Nature 355, 368-371 (1992)

13. Weinert, T. A. Radiat. Res. 132, 141-143(1992)

14. Weinert, T. A. \& Hartwell, L. H. Science 241, 317 (1988)

15. Kuerbitz, S. J., Plunkett, B. S., Walsh. W. V. \& Kastan M. B. Proc. natn. Acad. Sci. U.S.A. 89. 7491 (1992).

16. Kastan, M. B. et al. Cell 71, 587-597 (1992).
} 